\title{
In vitro and in vivo characteristics of frozen/thawed neonatal pig split-skin strips: A novel biologically active dressing for areas of severe, acute or chronic skin loss
}

\author{
ANNA CHIARINI, ILARIA DAL PRA and UBALDO ARMATO \\ Histology and Embryology Unit, Department of Biomedical and Surgical Sciences, \\ University of Verona Medical School, I-37134 Verona, Italy
}

Received August 10, 2006; Accepted October 6, 2006

\begin{abstract}
The recurrent shortage of human skin autografts or allografts used to close extensive wounds has rekindled the search for feasible alternatives. In the past, adult pig skin was a popular biological dressing, yet doubts regarding its benefits have induced most people to abandon its use. Here we investigated the aptness of neonatal pig split-skin (NPSS) strips to be used as a novel kind of temporary dressing for areas of skin loss. NPSS strips are able to be prepared in bulk amounts, stored at $-80^{\circ} \mathrm{C}$ for up to six months, and recovered by swift thawing at $45^{\circ} \mathrm{C}$ with no change in histological structure. When set into organ cultures in vitro for up to three weeks, these frozen/thawed NPSS strips exhibited both a skin-typical energy-linked metabolism (i.e., a predominant consumption of L-glutamine instead of glucose), and an enduring ability to secrete cytokines/ chemokines such as IL- $1 \alpha$, IL-6, GM-CSF, and TNF- $\alpha$; all features alike in quantitative terms to those exhibited by freshly prepared NPSS strips directly set into culture. Moreover, once applied as temporary dressings onto deep burn wounds in vivo, frozen/thawed NPSS strips produced, for at least seven days, porcine IL- $1 \alpha$, IL-6, GM-CSF, TNF- $\alpha$, and TGF- $\beta$; the cytokines/chemokines importantly involved in wound healing. Hence, frozen/thawed NPSS strips not
\end{abstract}

Correspondence to: Dr Ubaldo Armato, Histology and Embryology Unit, Department of Biomedical and Surgical Sciences, University of Verona Medical School, Strada Le Grazie 8, I-37134 Verona, Italy

E-mail: ubaldo.armato@univr.it

Abbreviations: CEA, cultured epithelial autografts; DPF, designated pathogen free; GM-CSF, granulocyte-macrophage colony stimulating factor; IC, immunocytochemistry; IL, interleukin; PERV, porcine endogenous retrovirus; TGF, tumour growth factor; TNF, tumour necrosis factor; WB, Western immunoblotting; NPSS, neonatal pigs split-skin

Key words: cultured epithelial autografts, neonatal pigskin, organ culture, xenografts, biological dressings, IL-1 $\alpha$, IL-6, GM-CSF, TNF- $\alpha$, TGF- $\beta$ only are capable of closing extensive areas of skin loss, but even release several cytokines/chemokines beneficial to tissue regeneration and repair.

\section{Introduction}

Extensive areas of human skin loss, e.g. burn wounds of $6,000-19,000 \mathrm{~cm}^{2}$ or more, are often difficult to cover with a suitable dressing. A burn must be closed swiftly and completely with a temporary, skin-like dressing (for an optimum result), to improve its clinical course (1). Autografts are the ideal cover for full-thickness burns, being easily revascularised and thus 'taking' with a high success rate without inducing any rejection reaction (1). Unfortunately, the size of available donor sites is inversely proportional to the extent of burn wounds (2), and technical refinements, such as repeated harvesting of donor sites and meshed autograft expansion, only partly solve the problem, while bringing in the dangers of local desiccation and infection (3). On the other hand, cultured epithelial autografts (CEAs) have given unpredictable and inconsistent clinical results because of low 'take' rates $(15-21 \%)$ due to non-optimal wound bed conditions (4), and second-set rejection responses evoked by the persistence of foreign (human or mouse) fibroblasts used as feeder layers (5). Allografts (or homografts) from living or dead donors are used by themselves or in association with autografts to cover extensive wounds (6). Allografts elicit earlier epithelisation and better end-results, but donor availability is limited by cultural and religious factors and by ongoing diseases such as tumours and infections. Repeated allograft applications trigger sensitisation and accelerated rejection of the second- or thirdset grafts, unless immunosuppressive therapy is used $(6,7)$. Moreover, allografting entails the risk of transmitting infectious agents to the host (8). The use of cultured allo-epithelial grafts is hampered by the same restraints mentioned for allografts and CEAs (5). The exploit of using xenografts in burn care was pioneered by Reverdin in the 19th century (9). Thenceforth, skin flaps taken from a number of animal species, such as the shark, frog, chicken, pigeon, rat, sheep, goat, cat, dog, and cow, have been used as biological dressings for sizeable skin wounds in animals and humans (10-15). Chemically-treated bovine skin (Dermodress ${ }^{\circledR}$ ) has also been applied as a long-term skin substitute onto deep 
burn wounds (16). However, just as happens for allografts (5), permanent transplantation of animal skin is precluded by immunologic rejection (17). During the past 40 years, adult pig skin in various forms (i.e. fresh, freeze-dried, preirradiated frozen, incorporating neomycin and/or silver ions, formaldehyde-fixed, glycerinated, lyophilised or nonlyophilised glutaraldehyde-fixed, and chlorhexidine-treated) has been by far the most popular xenograft used to cover burn wounds, donor sites, meshed autografts, areas of partial thickness skin loss, and non-healing cutaneous ulcers of various aetiology (18-29). Adult porcine skin apparently has all the features of an ideal wound protective cover, such as tensile strength; tissue adherence; flexibility; water-vapour permeability; heat retention; a human skin-like histological structure (18) unless deeply altered by chemical treatments; anti-bacterial and pain relieving effects; lack of antigenicity; no toxicity; low cost; and long shelf-life (18-29). Moreover, adult porcine skin xenografts were never vascularised $(15,18,20,30,31)$, even though being at times partly included within the healing tissue (32). Despite a slight cellular infiltration at the wound bed/xenograft boundary, no significant levels of anti-donor pig antibodies were induced and no immunological reaction or rejection evoked $(15,33)$. The pig skin dressing desiccated and detached from the wound bed, thereby acting more as a collagen prosthesis than as a true xenograft (34). However, the above mentioned advantages of the various forms of adult pig skin dressing were negated by several studies documenting that the removal of neomycin after reports of toxic and untoward reactions rendered porcine dressings ineffective; that there was no difference in the beneficial effects on wound healing observed between adult pig skin applied with mafenide acetate (Sulfamylon ${ }^{\circledR}$ ) cream and mafenide alone; that lyophilised porcine skin was not found to be more beneficial than petrolatum gauze or coarse mesh gauze changed thrice every day; that formalin-fixed pig skin behaved by far as the worst dressing; and finally, that adult pig skin did not confer any advantage over or protect any better than simple gauze (35-38). Hence, during the last two decades the use of adult pig skin has been declining steadily in clinical settings, being at present restricted to a few centres (39).

In this study, we examined the feasibility of using neonatal porcine split-skin (NPSS) strips, a novel kind of wound dressing. The results showed that frozen/thawed NPSS strips retain their viability, metabolism, and capacity for producing various cytokines/chemokines that play crucial roles in skin pathophysiology just as fresh NPSS strips do. Moreover, our findings also demonstrated that, once grafted onto severe burn wounds in vivo, frozen/thawed NPSS strips produced, for at least one week, several cytokines/chemokines, thereby acting both as a cover and as a biological dressing favouring the healing process.

\section{Materials and methods}

NPSS strip preparation and handling. All procedures employed for animal husbandry conditions, disease surveillance and screening procedures, and tissue banking standards were in keeping with Food and Drug Administration (FDA) guidelines (40) and the World Health Organisation
(WHO) (41). Neonatal piglets were from sows raised in a barrier facility and maintained free of designated pathogens (Designated Pathogen Free or DPF animals) via ad hoc surveillance programs and veterinary care. Piglets were directly washed in a warm antiseptic bath, anaesthetised with ethyl ether, and sacrificed by an intra-cardiac injection of $\mathrm{KCl}$ solution. Stripped hides were dipped into an antiseptic solution, stripped of hair, and put into RPMI-1640 medium (Sigma Italia S.p.A., Milan). After cleansing of subcutaneous fat lobules, the hides were cut into split-thickness (1.5-mmthick) strips (width, $7.5 \mathrm{~cm}$; mean length, 10-20 cm) using a dermatome (Nouvag AG, Goldach, Switzerland). Next, NPSS strips were incubated for $2 \mathrm{~h}$ in RPMI-1640 medium fortified with antibiotics and zidovudine (42). They were transferred to a 20\% glycerol-80\% RPMI-1640 medium and put into three sterile plastic envelopes that were heat-sealed and quarantined in a dedicated deep freezer. In parallel, samples taken from each piglet hide, NPSS strip and their respective soaking media underwent microbiological, mycological, and virological analyses. NPSS strips found to be sterile (average, 98\%) were transferred to an ad hoc deep freezer to be stored for up to 6 months. When needed, sterile frozen NPSS strips were taken out of this repository, rapidly thawed at $45^{\circ} \mathrm{C}$ (henceforth they will be referred to as frozen/ thawed strips), and washed with fresh RPMI-1640 medium to be either set into organ cultures in vitro or enclosed in three sterile heat-sealed envelopes sent by courier to the Burn Wound Care Unit (Major Hospital, Verona) to be grafted, as an ultimate supportive measure, onto patients suffering from severe burns. In five different instances, punch biopsies (4 mm in diameter) were taken from the frozen/thawed NPSS strips prior to (0-time), and 3 and 7 days after grafting in vivo to assess tissue production of various porcine cytokines/ chemokines.

In vitro organ cultures of NPSS strips. Both freshly prepared and frozen/thawed NPSS strips were cut into $2.5 \times 2.5 \mathrm{~cm}$ pieces that were cultured at $37^{\circ} \mathrm{C}$ inside the square wells of Falcon flasks in the presence of $95 \%$ air $/ 5 \% \mathrm{CO}_{2}$. Such organ cultures were kept for up to three weeks. The growth medium [RPMI medium fortified with antibiotics and 5\% ( $\mathrm{vol} / \mathrm{vol})$ adult horse serum (Cambrex Bio Science, Milan)] was changed with a fresh one every fourth day. Cell-conditioned media were stored at $-80^{\circ} \mathrm{C}$ to be subsequently analysed by biochemical means.

Metabolic assays of cultured NPSS strips. The uptake of L-glutamine was evaluated as follows. Twenty microliters of each NPSS strip-conditioned growth medium sample was incubated for $15 \mathrm{~min}$ at $37^{\circ} \mathrm{C}$ with $0.1 \mathrm{M}$ sodium acetate, $\mathrm{pH} 4.9$, in the presence of 0.05 units of glutaminase (EC 3.5.1.2) to convert the L-glutamine into glutamate and ammonium $\left(\mathrm{NH}_{3}\right)$. The resulting amount of ammonium was next determined by the reductive amination of $3.4 \mathrm{mM} 2$-oxoglutarate, using 12 units of L-glutamate dehydrogenase $(\mathrm{GLDH})$ and $0.23 \mathrm{mM}$ reduced NADPH in $0.1 \mathrm{M}$ sodium phosphate, $\mathrm{pH}$ 7.4. Finally, the decrease in absorbance at $340 \mathrm{~nm}$, due to the oxidation of NADPH, was quantified, and this was proportional to the concentration of the L-glutamine remaining in the cell-conditioned medium samples. The 
utilisation of D-glucose was appraised in the NPSS stripconditioned growth medium samples by a glucose oxidase assay according to Trinder et al (43) (Sigma). The lactic acid released into the NPSS strip-conditioned growth medium samples was assessed via a lactate oxidase assay according to Barham et al (44), again as developed by Sigma. The urea secreted into the NPSS strip-conditioned growth medium samples was assessed via a specific commercial kit according to the manufacturer's instructions (Sigma). In each of the above instances as well as in the case of the secretion of various cytokines/chemokines (see below), cumulative curves were constructed from the data pertaining to fresh or frozen/thawed NPSS strips. Each experiment was performed at least five times.

ELISA assays of porcine cytokines/chemokines released by cultured NPSS strips. Growth medium samples conditioned by contact with either freshly prepared or frozen/thawed NPSS strips were assayed for a set of porcine cytokines/ chemokines by using antibodies not cross-reacting with the corresponding human cytokines. A commercial ELISA kit was used to assay IL-6 (R\&D Systems Inc., Minneapolis, $\mathrm{MN})$. Moreover, we used various ELISA tests developed in our laboratory to assay IL- $1 \alpha$, GM-CSF, TGF- $\beta$, and TNF- $\alpha$. For each ELISA a 96-well plate was coated with $50 \mu 1$ of growth medium diluted $1: 1$ in $0.1 \mathrm{M}$ carbonate buffer $\mathrm{pH} 9.0$, overnight at $4^{\circ} \mathrm{C}$, and then blocked with $200 \mu \mathrm{l}$ of PBS/1\% bovine serum albumin (BSA) for $1 \mathrm{~h}$ at room temperature. After washing with PBS/0.05\% Tween solution, a polyclonal goat or rabbit antibody specific for porcine IL-1 $\alpha$, GM-CSF, TGF- $\beta$, and TNF- $\alpha$ (R\&D Systems Inc.) was added at a concentration of $1 \mu \mathrm{g} / \mathrm{ml}$ in PBS/5\% BSA and incubated for $1 \mathrm{~h}$ at room temperature. After removal of excess antibody, an appropriate second antibody conjugated to horseradish peroxidase (Santa Cruz Biotechnology, Inc., Santa Cruz, CA) diluted 1:1000 in PBS/5\% BSA was added. Following a wash procedure to remove any unbound antibody, the chromogen substrate tetramethylbenzidine (TMB) solution was added to the wells and the enzymatic colorimetric reaction was stopped by adding $100 \mu \mathrm{l}$ of $0.1 \mathrm{M} \mathrm{H}_{2} \mathrm{SO}_{4}$ to each well. The absorbance was read at $450 \mathrm{~nm}$ having blanked the plate reader against a chromogen blank composed of $100 \mu \mathrm{l}$ each of TMB solution and stop solution. For each ELISA a standard curve was generated using recombinant porcine cytokines/chemokines (R\&D Systems Inc.). The detection limits of these assays were: IL-1 $\alpha, 0.16$ ng/well; GM-CSF, 0.2 ng/well; TGF- $\beta, 1.6$ ng/well; TNF- $\alpha, 0.5 \mathrm{ng} /$ well; and IL-6, $10 \mathrm{pg} / \mathrm{ml}$. The results were expressed as secretion values per triplicate wells per 8-10 distinct experiments.

Histology and immunocytochemistry (IC). Samples of freshly prepared or frozen/thawed NPSS strips and biopsies of frozen/thawed NPSS strips taken just prior to grafting (experimental 0-time) in vivo or 3 and 7 days after grafting were instantly frozen using precooled $\left(-80^{\circ} \mathrm{C}\right)$ isopentane in liquid nitrogen (45). Next, sections were cut at 5-to $15-\mu \mathrm{m}$ thicknesses in a 1720 Digital cryostat (Leitz Lauda, Milan, Italy), mounted on aminopropyl-triethoxysilane (APES)coated slides, fixed for $20 \mathrm{~min}$ in acetone at $-20^{\circ} \mathrm{C}$, and dried (45). Tissue sections were subsequently stained with Mayer's haematoxylin and eosin (H\&E) (46). The procedures followed for IC were those described by Jackson and Blythe (45). Tissue sections were washed twice with PBS containing BSA $(1.0 \% \mathrm{w} / \mathrm{v})$ and $\mathrm{NaN}_{3}(0.1 \% \mathrm{w} / \mathrm{v})$, and incubated for $60 \mathrm{~min}$ at room temperature with primary antibodies (at $1.0 \mu \mathrm{g} \mathrm{ml}^{-1}$ ) specifically directed against porcine (no human crossreaction) IL-1 $\alpha$, IL-6, GM-CSF, and TNF- $\alpha$ (R\&D Systems Inc.). Next, tissue sections were washed thrice with PBSBSA (1\%) solution and incubated for an additional $1 \mathrm{~h}$ at room temperature with proper second antibodies (1:100 dilution) conjugated with alkaline phosphatase (all from Santa Cruz Biotechnology). Specific IC colours were developed with Fast TR naphtol or Fast Red AS-MX tablet sets (both from Sigma). Control tissue sections were run in parallel, the primary or secondary antibody being omitted from the procedure. Specimens were examined under an Olympus BX-60 microscope and photographed with an Olympus $3300^{\mathrm{TM}}$ digital camera.
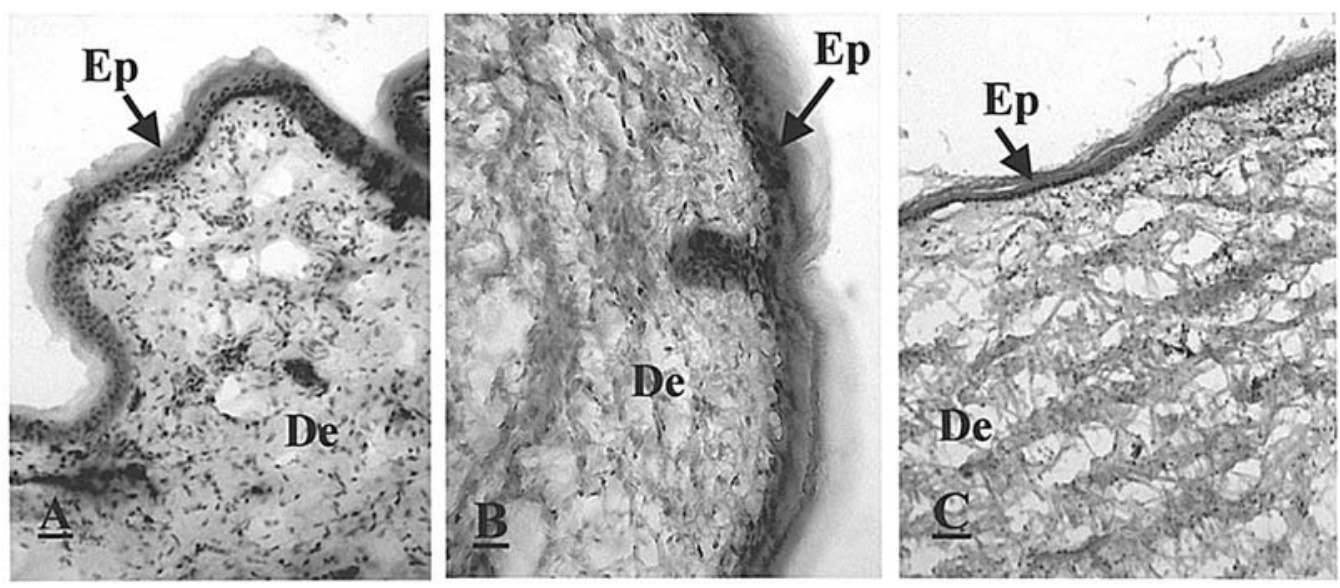

Figure 1. The histological structure of processed NPSS strips as seen in 5- to 8- $\mu$ m-thick cryostat sections. For technical details, see the Materials and methods section. (A) Freshly prepared NPSS strip. (B) Frozen/thawed NPSS strip; note the well-preserved structure of the tissue compared to the fresh tissue shown in A. (C) A sample of frozen (for three months at $-80^{\circ} \mathrm{C}$ )/thawed NPSS strip set into an in vitro organ culture for 16 days. The epidermal cell layers are still distinct and a visible stratum corneum indicates that the epidermis is undergoing normal differentiation/apoptosis. The structure of the cultured dermis is similar to that of specimens A and B save for a minor degree of oedema. H\&E staining. Magnification, x40. Ep, epidermis; De, dermis. 

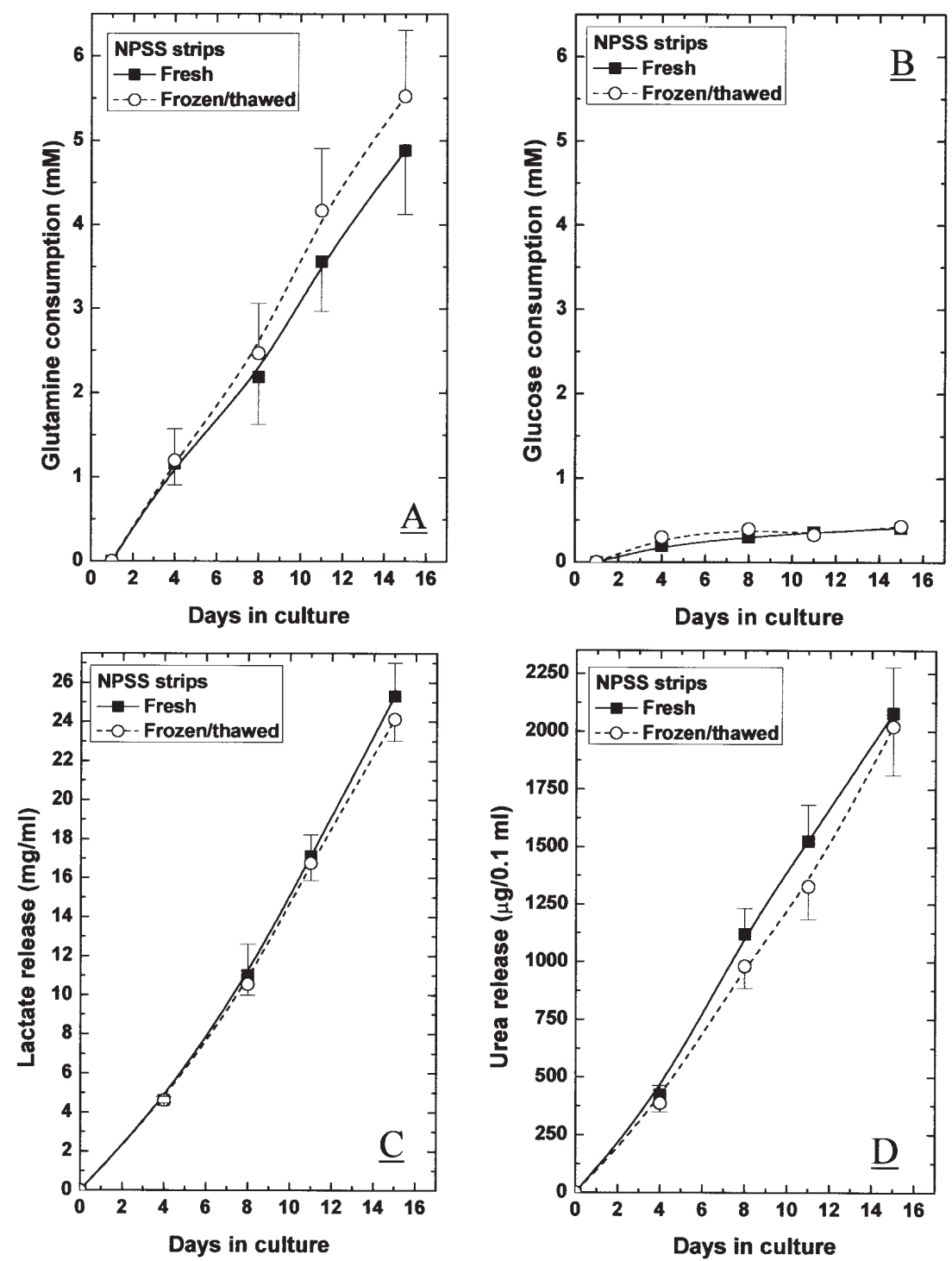

Figure 2. Energy-linked metabolic processes similarly occur in fresh and in frozen/thawed NPSS strips set into organ cultures in vitro. (A) The similar consumption of L-glutamine by fresh and by frozen/thawed NPSS strips shows that this amino acid is, in both instances, as in vivo, the main energy-producing fuel (49). (B) D-glucose is only marginally taken-up by fresh and by frozen/thawed NPSS strips. (C) The comparable release of lactate into the RPMI-1640 medium by fresh and by frozen/thawed NPSS strips. In the skin in vivo, lactate is a by-product of L-glutamine and D-glucose metabolisms (49). (D) The release of urea into the RPMI-1640 medium by fresh and by frozen/thawed NPSS strips is quite similar, revealing an ongoing turnover of amino acids and proteins. Points on the curves are the means \pm SEM of triplicate wells from 8-10 distinct experiments for each group of samples. No significant statistical difference was obtained between the mean values of time-corresponding samples.

Western immunoblotting (WB). Extraction of total proteins from punch biopsies (diameter, $4 \mathrm{~mm}$ ) of frozen/thawed NPSS strips was carried out just prior to grafting in vivo (experimental 0 -time) and 3 and 7 days later. The skin samples were washed with cold PBS and homogenised in $\mathrm{T}_{-} \mathrm{PER}^{\mathrm{TM}}$ tissue protein extraction reagent (Pierce Chemical Co., Rockford, IL) containing a complete EDTA-free protease inhibitor cocktail (Roche Diagnostics S.p.A., Monza, Italy). Subsequent procedures for WB were carried out according to Chiarini et al (47). The protein contents of the samples were assayed by Bradford's method (48) using bovine serum albumin as a standard. Equal amounts (25-30 $\mu \mathrm{g}$ ) of proteins from each sample were boiled in sample buffer $(0.0625 \mathrm{M}$ Tris- $\mathrm{HCl}, \mathrm{pH} 6.8,2 \% \mathrm{w} / \mathrm{v}$ SDS, $5 \% \mathrm{w} / \mathrm{v}$ ßmercaptoethanol, $10 \% \mathrm{v} / \mathrm{v}$ glycerol, and $0.002 \% \mathrm{w} / \mathrm{v}$ bromphenol blue) and electrophoresed in $12 \% \mathrm{w} / \mathrm{v}$ SDSpolyacrylamide gel. The separated proteins were blotted onto a nitrocellulose membrane $(0.45 \mu \mathrm{m}$; Pall Life Sciences, Ann Arbor, MI). To immunodetect the porcine cytokines/ chemokines IL- $1 \alpha$, IL-6, GM-CSF, TNF- $\alpha$ and TGF- $\beta$, the blots were probed with specific goat or rabbit IgG polyclonal antibodies used at a final dilution of $1.0 \mu \mathrm{g} \mathrm{ml}^{-1}$; (all from R\&D Systems Inc. except the anti-IL-6 antibody from BioSource International Inc., Camarillo, CA). Blots were next incubated with alkaline phosphatase-conjugated antigoat or anti-rabbit IgGs (Santa Cruz Biotechnology, Inc.), and stained with BCTP/NBT liquid substrate reagent (Sigma). Developed blots were scanned in an Odyssey ${ }^{\circledR}$ Infrared imaging system (Li-Cor Biosciences Inc., Lincoln, $\mathrm{NE}$ ) and the integrated intensity of each specific protein band was accurately quantified using proprietary software.

Statistical analysis. The Student's t-test was used to compare mean values and a significance level of 0.05 was chosen. 

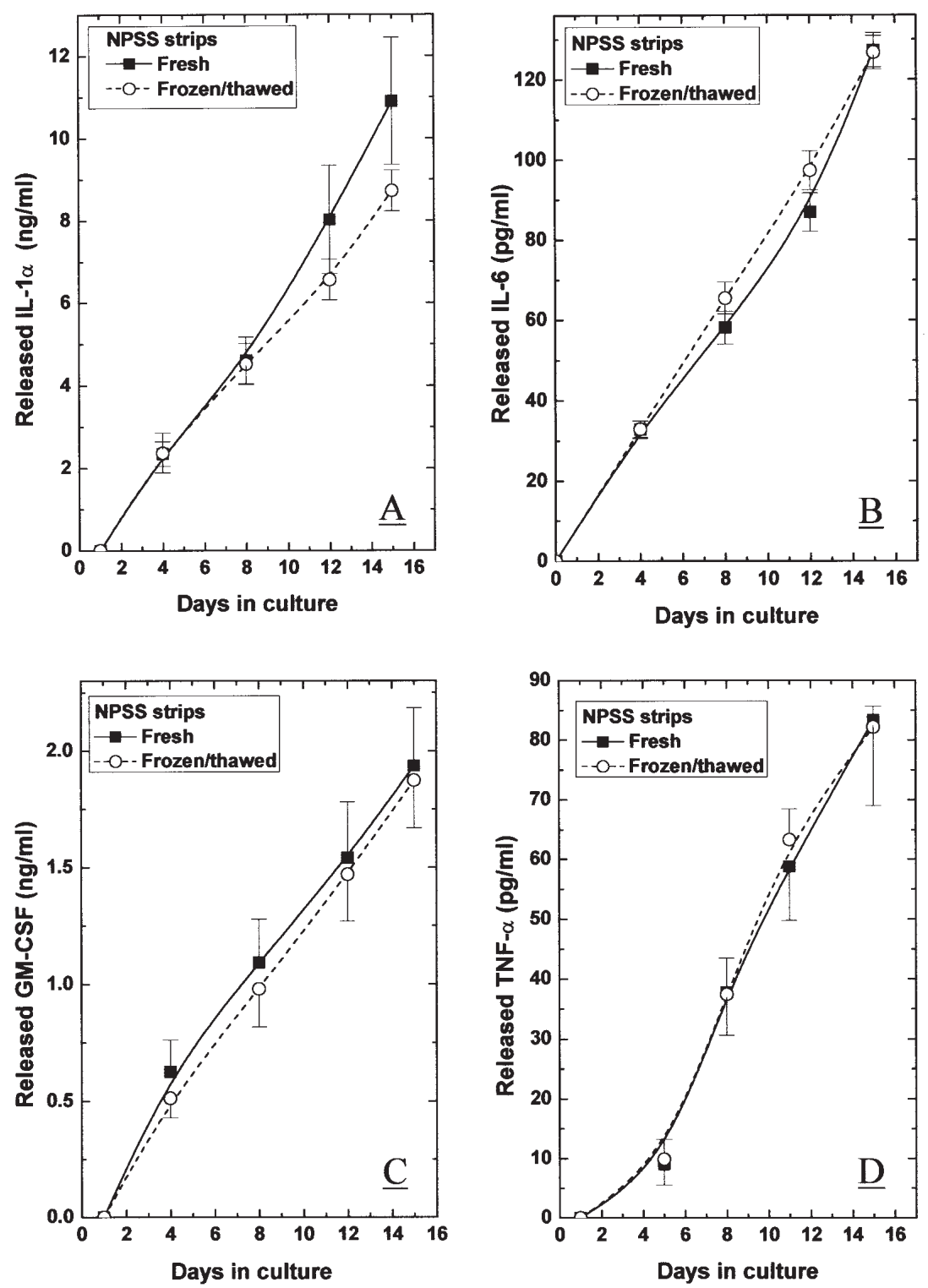

Figure 3. Secretion of the various porcine cytokines/chemokines into the RPMI-1640 medium is similarly upheld by fresh and by frozen/thawed NPSS strips set into organ cultures in vitro. Similar amounts of IL-1 $\alpha$ (A), IL-6 (B), GM-CSF (C), and TNF- $\alpha$ (D) were released with time by the two groups of specimens. The assay procedures employed are detailed in the Materials and methods section. Points on the curves are the means \pm SEM of triplicate wells from $8-10$ distinct experiments for each group of samples. No significant statistical difference was obtained between the mean values of time-corresponding samples.

\section{Results}

NPSS strips can be prepared in bulk amounts (e.g. 1.5-2.0 $\mathrm{m}^{2}$ from 15-20 piglets) by two workers in the course of a single day. Our first endeavour was to establish whether the laboratory procedures required to produce frozen/thawed NPSS strips would significantly alter their biological characteristics (e.g. histology, energy metabolism, and cytokine/chemokine release) compared to those exhibited by fresh NPSS strips.

Observations carried out under a light microscope of H\&E-stained cryostat sections of frozen/thawed NPSS strips revealed a well preserved histological structure, which compared with those of the fresh strips (Fig. 1A and B).

Moreover, analysis of cryostat sections of frozen/thawed NPSS strips revealed that, after two-three weeks of staying in organ cultures in vitro, the epidermal layers were still well preserved and were undergoing the expected differentiation/ apoptosis (Fig. 1C), while the dermal connective tissue was slightly oedematous (Fig. 1A and C).

These histological findings were in keeping with the results of biochemical assays showing that frozen/thawed NPSS strips could, in the course of 15 days in organ culture, both maintain a skin-specific energy metabolism and secrete various cytokines/chemokines at levels not significantly different from those exhibited by fresh strips directly set into culture. As shown in Fig. 2, cultured, both fresh and frozen/ thawed NPSS strips similarly elected L-glutamine (Fig. 2A) as their principal energy supply instead of D-glucose (a typical skin metabolic feature) (Fig. 2B) (49) and continued releasing like amounts of two catabolites, lactic acid and urea (Fig. 2C and D, respectively) into the growth medium.

To note, once set into organ cultures in vitro, both freshly prepared and frozen/thawed NPSS strips continuously 

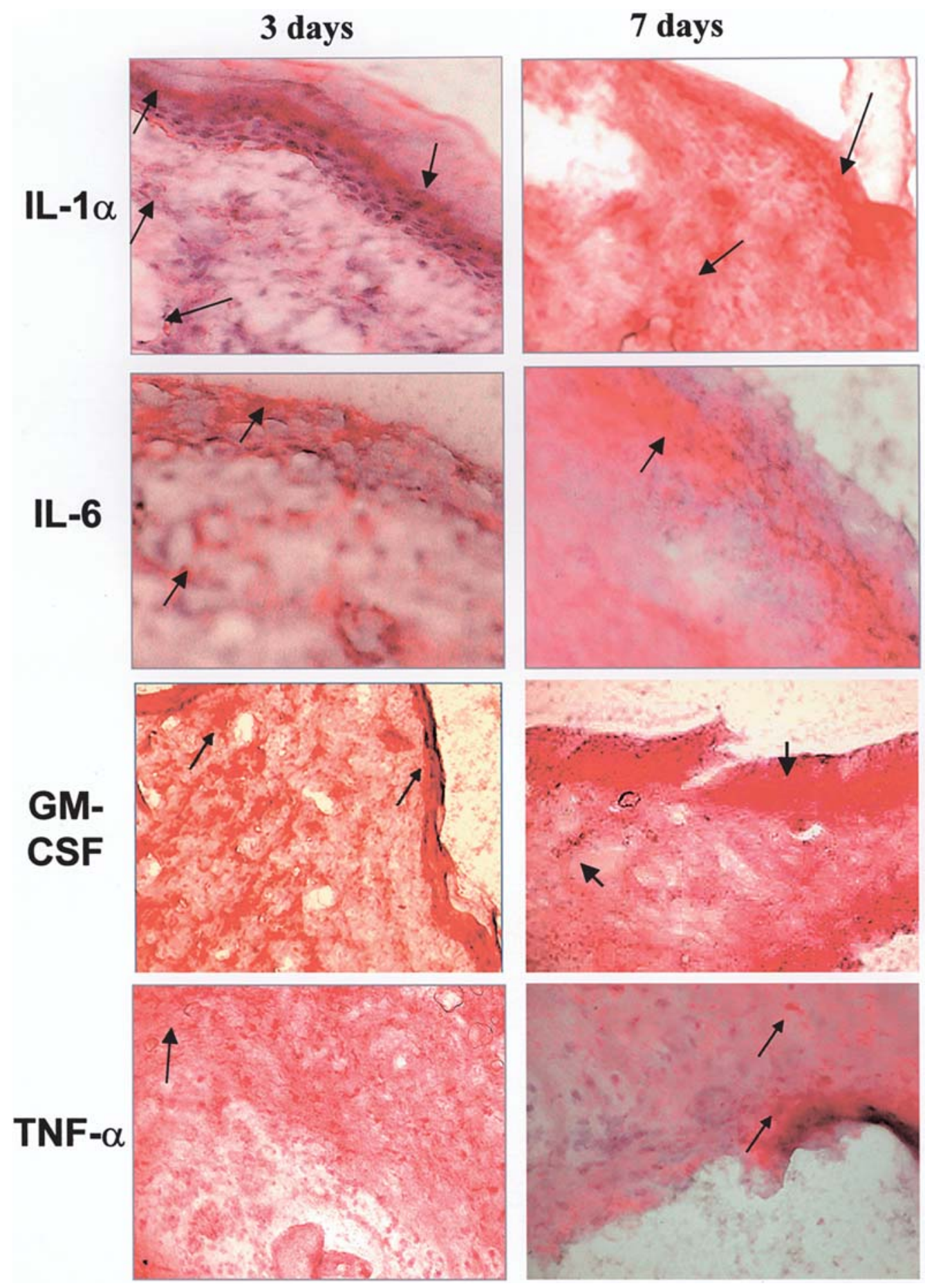

Figure 4. Frozen/thawed NPSS strips produce immunocytochemically detectable amounts of various porcine cytokines/chemokines after 3 and 7 days of grafting onto deep burn wounds in vivo. Note the increased intensity of the antibody decoration (in pink and red; arrows) in 7-day samples vs 3-day specimens for each cytokine/chemokine examined. The specific primary antibodies used did not cross-react with the corresponding cytokines of human origin. A light haematoxylin counterstaining reveals that the histological structure of the grafted NPSS strips is still well-preserved even after 7 days of grafting in vivo. Original magnification, $\mathrm{x} 40$ or $\mathrm{x} 100$.

secreted reciprocally matching amounts of four porcine cytokines/chemokines, IL-1 $\alpha$, IL-6, GM-CSF, and TNF- $\alpha$, all of which are known to play relevant roles in skin physiopathology (50) (Fig. 3). Conversely, TGF- $\beta$ was undetected in the sampled conditioned growth media (not shown).

Collectively, these findings showed that frozen/thawed NPSS strips did not differ from fresh NPSS strips in histological structure, energy metabolism, and the ability to release assayable amounts of the cytokines/chemokines.
The second aim of the present study was to establish whether, once grafted onto deep burn wounds in vivo, frozen/ thawed NPSS strips would remain biologically active for a long period of time or rapidly become inert dressings. The results of the immunocytochemical and Western blot analyses support the former alternative. Notably, at 0-time (i.e. just prior to grafting) only porcine IL-6 was expressed by the frozen/thawed NPSS strips (Fig. 5B). However, three days after grafting in vivo, porcine IL-1 $\alpha$, IL-6, GM-CSF, and TNF- $\alpha$ were distinctly expressed by the deeper epidermal 

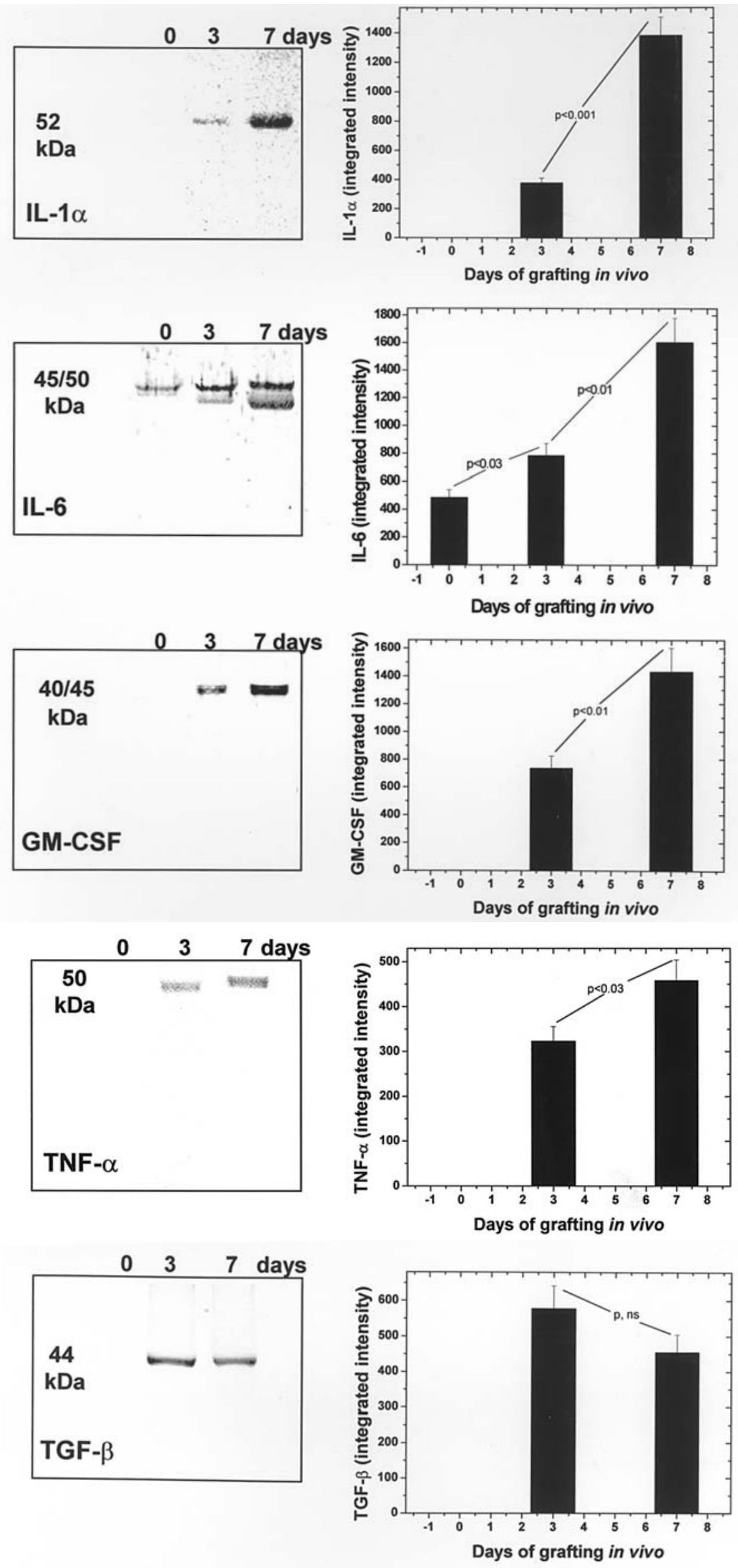

Figure 5. (Left panels) WB analysis of total protein extracts from biopsy specimens shows that frozen/thawed NPSS strips still produce various porcine cytokines/chemokines after 3 and 7 days of grafting onto deep burn wounds in vivo. The immunoblots shown are typical for each cytokine investigated. (Right panels) Densitometric evaluation of the specific protein bands for each cytokine. Bars are \pm SEM of five distinct biopsies. The specific primary antibodies used for WB did not cross-react with the corresponding cytokine of human origin. 
layers and by sparse dermal cells (Fig. 4). In addition, 7 days after grafting in vivo, the expression of the same four porcine cytokines/chemokines became increasingly more intense in both the epidermal and dermal layers (Fig. 4). Light counterstaining with haematoxylin of the IC specimens showed that the histological structure of the frozen/thawed NPSS strip biopsies was well-preserved at day 3 and 7 of grafting in vivo (Fig. 4).

Analysis by WB of the total protein extracts of the biopsies from the frozen/thawed NPSS strips grafted onto burn wounds in vivo substantiated the immunocytochemical findings (Fig. 5). Only porcine IL-6 was expressed by the frozen/thawed NPSS strips prior to grafting (at 0-time) (Fig. 5B). Frozen/thawed NPSS strips at grafting day 3 produced discrete amounts of IL-1 $\alpha$, IL-6, GM-CSF, and TNF- $\alpha$. Densitometric analysis of the specific protein bands showed that the expression of these cytokines was significantly more intense by day 7 after grafting in vivo: IL- $1 \alpha,+275 \%, \mathrm{p}<0.001$; IL-6, +104\%, p<0.01; GM-CSF, $+100 \%, \mathrm{p}<0.01$; and TNF- $\alpha,+42 \%, \mathrm{p}<0.03$ (Fig. 5). IL-6 expression was also greater at day 3 vs day $0(+66 \%$, $\mathrm{p}<0.03)$. On the other hand, the expression of TGF- $\beta$ was absent at 0 -time, but detectable by day 3 and not significantly changed by day 7 of grafting in vivo (day 7 vs day $3,-21 \%$, p not significant) (Fig. 5). Therefore, frozen/thawed NPSS strips can resume and even increase or maintain the production of certain cytokines/chemokines for at least 7 days after being grafted onto burn wounds in vivo.

\section{Discussion}

The present findings show that frozen/thawed NPSS strips maintain their viability for long periods, as attested by their normal histological structure and their specific metabolic capabilities, both in organ cultures in vitro and after grafting onto burn wound beds in vivo. Consequently, they have all the properties needed to perform both as ideal covering bandages $(1,2)$ and as biologically active wound dressings. The latter capability is not shared by the various chemicallyor radiation-treated, hence biologically inert adult pig skin dressings commercially available and clinically used both in the past and at present (18-29).

In fact, when temporarily grafted in vivo, frozen/thawed NPSS strips not only close the burn wound, thereby preventing the loss of significant amounts of water, electrolytes, and precious proteins, blocking the penetration of foreign infectious micro-organisms, and reducing pain (and the need for analgesic medications), but even favour the regenerative and healing processes via the production and extracellular release of a complex set of beneficial cytokines/ chemokines (50). The preliminary clinical results of grafting frozen/thawed NPSS strips onto severely burnt patients have consistently confirmed the favourable effects of such biologically active dressings, used by themselves or in combination with underlying meshed skin autografts, on wound healing in the complete absence of any adverse side effects and of any rejection or inflammatory reactions (manuscript in preparation).

An important advantage from the practical standpoint is that frozen/thawed NPSS strips can be prepared in large amounts, checked for sterility, stored in a deep freezer, and when required promptly made ready for grafting in vivo. This means that, at short notice, even an adult patient with $95 \%$ burns, a surface of $1.7 \mathrm{~m}^{2}$ or more, can be covered with frozen/ thawed NPSS strips. Moreover, when used alone or in conjunction with meshed skin autografts or allografts, these same strips may benefit even split-skin graft donor sites $(11,20)$, chronic skin ulcers of any aetiology, and other pathological skin conditions such as toxic epidermal necrolysis (51).

Here, it must also be stressed that, at variance with allografts, especially those from cadavers, frozen/thawed NPSS strips are endowed with highly reproducible long-term biological properties both in in vitro cultures and, most important, after grafting in vivo. Conceivably, the wound bed fluid infiltrates the grafted NPSS strips maintaining their hydration and providing nutrients for at least a week before desiccation, avascular necrosis, and spontaneous detachment from the wound bed ensue. Thus, the grafted frozen/thawed NPSS strips not only continue preferring L-glutamine as the main energy-producing fuel, a specific feature of skin (49), but even continue synthesizing and secreting cytokines/ chemokines that play key roles in skin pathophysiology (50). Reportedly, IL- $1 \alpha$ is importantly involved in inflammatory skin conditions and in epidermal cell growth and repair processes via members of the IL-1R (receptor) family $(52,53)$. IL-6 is a multifunctional cytokine of the haemopoietin family comprising amongst others the granulocyte-colony stimulating factor (G-CSF) and acting via the gp130 signaltransducing subunit (54). IL-6 operates as a growth factor for keratinocytes either via its specific receptor signalling (55) and/or by inducing the production and release of keratinocyte growth factor (KGF) by dermal fibroblasts (56). By means of paracrine and autocrine receptor-mediated loops, IL- 6 also may enhance the proliferation of human adult dermal fibroblasts and/or up-regulate the production of collagen, glycosaminoglycans (GAGs), interstitial collagenase, and stromelysin-1 by such cells, thereby favouring the inflammatory processes related to wound healing (57). On its own part, the haemopoietin GM-CSF (54) is a pleiotropic cytokine secreted by keratinocytes, fibroblasts, endothelial cells, dendritic cells, macrophages, and lymphocytes; cell types involved in wound healing. GM-CSF plays complex roles like accelerating re-epithelisation, enhancing neovascularisation, increasing the formation of granulation tissue, and inducing secondary cytokines such as IL-6, TGF-B, and IFN- $\gamma$ (58). Via its ubiquitous receptors, TNF- $\alpha$ is involved in various relevant aspects of skin wound healing such as cell proliferation, cell differentiation, inflammation, and immunomodulation (59, and refs. therein). Finally, the members of the TGF- $\beta$ family are pleiotropic cytokines that i) orchestrate a large number of cellular activities related to wound healing and tissue fibrosis via specific receptors and Smad-protein-regulated intracellular signalling cascades in keratinocytes, fibroblasts, endothelial cells and monocytes, and ii) modulate dermal-epidermal interactions in wound repair (60, and refs. therein). To note, the pro-fibrotic activity of TGF- $\beta$ which is mediated via the induction of connective tissue growth factor (CTGF) expression in fibroblasts, is counteracted by TNF- $\alpha$ (61). Therefore, just as 
in the case of whole skin or cultured epithelial autografts and allografts, frozen/thawed NPSS grafts in vivo may work not only as mechanical bandages, but even as pharmacologic tools providing growth factors and cytokines/chemokines that accelerate regeneration/repair processes (62). Examples of human cells responding to porcine cytokines have been reported (63).

It should be recalled here that using living human skin allografts entails the risk of transmitting infectious agents to patients, particularly viruses such as the Epstein-Barr virus, cytomegalovirus, HIV, Kaposi-sarcoma-associated herpes virus and hepatitis viruses (8,63-66), as well as prions (67). Such risks are attenuated, but not entirely removed, by procedures that also do away with allograft viability (68). On the other hand, transmission of xenozoonoses (xenoses) by grafting living pig tissues or organs is partly restricted by using DPF animals undergoing adequate surveillance programs and veterinary care (69). The infectivity of porcine viruses, especially endogenous retroviruses (PERVs), for humans in vivo is still an object of controversy. However, it must be stressed that all published retrospective studies on human patients or subjects exposed to living pig tissues (e.g. following the grafting of pig islet cells or fetal neurons, or vascular connections to extra-corporeal pig livers or kidneys, or such as butchers who are in constant contact with fresh pig tissues and blood) have failed to demonstrate the presence of PERV DNA or RNA sequences or the occurrence of a PERV seroconversion in the hosts or exposed workers (71-85). Conversely, it has been proven that natural antibodies prevent the transmission of PERV to human cells in vivo (81). Furthermore, no evidence exists that PERVs infect other mammalian species, including nonhuman primates $(80,84)$. Nevertheless, adequate caution, like the exposure of NPSS strips to zidovudine (42), and patient follow-up are in order for the temporary grafting of frozen/thawed NPSS strips onto wounds in vivo. Additional procedures, such as the inhibition of PERV expression by RNA interference or the remodelling of the PERV envelope glycoprotein (86-88), will further ensure the safety of using frozen/thawed NPSS strips in clinical settings.

\section{References}

1. Fisher JC: Skin - the ultimate solution for the burn wound. N Engl J Med 311: 466-467, 1984.

2. Song IC, Bertram BE, Mohn MP and Koehnlein E: Heterografts as biological dressings for large skin wounds. Surgery 59: 576-583, 1966.

3. Gallico GG III: Biologic skin substitutes. Clin Plast Surg 17: 519-526, 1990.

4. Blight A, Mountford EM, Cheshire IM, Clancy JM and Levick PL: Treatment of full skin thickness burn injury using cultured epithelial grafts. Burns 17: 495-498, 1991.

5. Hultman CS, Brinson GM, Siltharm S, deSerres S, Cairns BA, Peterson HD and Meyer AA: Allogeneic fibroblasts used to grow cultured epidermal autografts persist in vivo and sensitize the graft recipient for accelerated second-set rejection. J Trauma 41: 51-58, 1996.

6. Miller TA, Switzer WE, Foley FD and Moncrief JA: Early homografting of second-degree burns. Plast Reconstr Surg 40: 117-125, 1967.

7. Delmonico FL, Cosimi AB and Russell PS: Temporary skin transplantation for the treatment of extensive burns. Ann Clin Res 13: 373-381, 1981.

8. Clarke JA: HIV transmission and skin grafts. Lancet 1: 983 , 1987.
9. Reverdin JL: Greffe epidermiques. Bull Soc Imperiale Chir 10: 483-511, 1869.

10. [No authors listed]: Making skin from sharks: early tests offer new hope for burn victims. Time 117: 53, 1981.

11. Zhang YH: Use of sheep and goatskin as biological dressing in the treatment of burns. Chung Hua Wai Ko Tsa Chih 22: 295-296, 1984.

12. Silvetti AN, Cotton C, Byrne R, Berrian JH and Fernandez Mendez A: Preliminary experimental studies of bovine embryo skin grafts. Transplant Bull 4: 25-26, 1957.

13. Switzer WE, Moncrief JA, Mills W, Order SE and Lindberg RB: The use of canine heterografts in the therapy of thermal injuries. J Trauma 6: 391-398, 1966 .

14. Piccolo N, Piccolo-Lobo N, Piccolo-Daher M, et al: Use of frogskin as a temporary biological dressing. Proc Am Burn Assoc p24, 1992.

15. Sokolic IH, Farpour A, Ulin AW and Howard J: The use of heterograft skin as a biological dressing. Surg Forum 10: 847-849, 1960.

16. Kon M, Sagi A and Mahler D: Dermodress: a temporary skin substitute for coverage of exposed vital structures in the hand. Ann Plast Surg 20: 136-139, 1988.

17. Auchincloss $\mathrm{H} \mathrm{Jr}$ and Sachs DH: Xenogeneic transplantation. Annu Rev Immunol 16: 433-470, 1998.

18. Bromberg BE, Song IC and Mohn MP: The use of pig skin as a temporary biological dressing. Plastic Reconstr Surg 36: 80-90, 1965.

19. Wood M and Hale HW Jr: The use of pigskin in the treatment of thermal burns. Am J Surg 124: 720-723, 1972.

20. Chang WHJ, Gomez NH and Edelstein M: Use of lyophilised pigskin for donor site cover. Br J Plast Surg 26: 147-149, 1973.

21. Silverstein P: Evaluation and preparation of formalin-fixed cutaneous grafts as a temporary wound cover for burned soldiers. US Army Institute of Surgical Research Annual Progress Report, FY, BAMC, Fort Sam, Houston, TX, Section 34, 1972 .

22. Elliott RA Jr and Hohen JG: Use of commercial porcine skin for wound dressings. Plast Reconstr Surg 52: 401-405, 1973.

23. Kaisary AV: A temporary biological dressing in the treatment of varicose ulcers and skin defects. Postgrad Med J 53: 672-673, 1977.

24. Morris DM, Hall GM and Elias EG: Porcine heterograft dressings for split-thickness graft donor sites. Surg Gynecol Obstet 149: 893-894, 1979.

25. Basile AR: A comparative study of glycerinized and lyophilized porcine skin in dressings for third-degree burns. Plast Reconstr Surg 69: 969-974, 1982.

26. Zhu DA and Cao QD: Chlorhexidine (hibitane)-alcohol refrigerated porcine skin - a simple and long-term stored xenograft. Chung Hua Cheng Hsing Shao Shang Wai Ko Tsa Chih 2: 203-206, 1986.

27. Mu XX: Treatment of full-thickness burn by planned replacement using glutaraldehyde porcine skin with an autograft. Chung Hua Cheng Hsing Shao Shang Wai Ko Tsa Chih 5: 193-196, 1989.

28. Tang ZY: Irradiated porcine skin in the treatment of seconddegree burn. Chung Hua Cheng Hsing Shao Shang Wai Ko Tsa Chih 6: 187-188, 1990.

29. Li G, Cao Y and Wu X: Experimental study of radiated pigskin incorporated with norfloxacin and silver. Chung Hua Cheng Hsing Shao Shang Wai Ko Tsa Chih 10: 206-208, 1994.

30. Aronoff M, Fleishman P and Simon DL: Experience in the application of porcine xenografts to split-graft donor sites. J Trauma 16: 280-283, 1976.

31. Salisbury RE, Wilmore DW, Silverstein P and Pruitt BA Jr: Biological dressings for skin graft donor sites. Arch Surg 106: 705-706, 1973.

32. Lawrence JC: Dressings for burns. In: Principles and Practice of Burn Management. Settle J (ed). Churchill Livingstone, New York, pp259-269, 1996.

33. Rappaport I, Pepino AT and Dietrick W: Early use of xenografts as a biologic dressing in burn trauma. Am J Surg 120: 144-148, 1970.

34. Burd A: Glycerolised allogenic skin: transplant or dressing? A medico-legal question. Burns 28 (suppl 1): 34-39, 2002.

35. Habal MB: (Editorial) On the cutaneous porcine heterograft. Plast Reconstr Surg 57: 367-368, 1976.

36. German JD, Wooley TE, Achauer B, Fornas DW and Bartlett RH: Porcine xenograft burn dressings. A critical reappraisal. Arch Surg 104: 806-808, 1972. 
37. Breach NM, Davies DM and Yiacoumettis A: Study of effects of porcine skin and bovine dermis on the healing of split-skin graft donor sites in humans. Plast Reconstr Surg 63: 546-549, 1979.

38. Levine NS, Lindberg RA, Salisbury RE, Mason AD Jr and Pruitt BA Jr: Comparison of coarse mesh gauze with biologic dressings on granulating wounds. Am J Surg 131: 727-729, 1976.

39. Chiu T and Burd A: 'Xenograft' dressing in the treatment of burns. Clin Dermatol 23: 419-423, 2005.

40. Department of Health and Human Services, Public Health Service, Draft Public Health Service (PHS): Guideline on Infectious Disease Issues in Xenotransplantation. Federal Register 61: 49920-49932, 1996.

41. World Health Organization (WHO): Xenotransplantation: Guidance on Infectious Disease Prevention and Management. WHO, Geneva, Switzerland, 1998.

42. Qari SH, Magre S, Garcia-Lerma JG, Hussain AI, Takeuchi Y, Patience C, Weiss RA and Heneine W: Susceptibility of the porcine endogenous retrovirus to reverse transcriptase and protease inhibitors. J Virol 75: 1048-1053, 2001.

43. Trinder P: Determination of blood glucose using an oxidaseperoxidase system with a non-carcinogenic chromogen. J Clin Pathol 22: 158-161, 1969.

44. Barham D and Trinder P: An improved colour reagent for the determination of blood glucose by the oxidase system. Analyst 97: 142-145, 1972.

45. Jackson $\mathrm{P}$ and Blythe D: Immunolabelling techniques for light microscopy. In: Immunocytochemistry: A Practical Approach. Beesley JE (ed). IRL Press at Oxford University Press, Oxford, pp15-41, 1995.

46. Bancroft JD and Cook HC: Manual of Histological Techniques and their Diagnostic Application. Churchill Livingstone, Edinburgh, p27 and pp243-244, 1994.

47. Chiarini A, Whitfield JF, Armato U and Dal Pra I: Protein kinase $C-\beta_{\text {II }}$ is an apoptotic lamin kinase in polyomavirustransformed, etoposide-treated pyF111 rat fibroblasts. J Biol Chem 277: 18827-18839, 2002. Epub March 18, 2002.

48. Bradford MM: A rapid and sensitive method for the quantitation of microgram quantities of protein utilizing the principle of protein-dye binding. Anal Biochem 72: 248-254, 1976.

49. Zielke HR, Ozand PT, Tildon JT, Sevdalian DA and Cornblath M: Reciprocal regulation of glucose and glutamine utilization by cultured human diploid fibroblasts. J Cell Physiol 95: 41-48, 1978.

50. Holman DM and Kalaaji AN: Cytokines in dermatology. J Drugs Dermatol 5: 520-524, 2006.

51. Green D, Law E and Still JM: An approach to the management of toxic epidermal necrolysis in a burn centre. Burns 19: 411-414, 1993.

52. Dinarello CA: The biological properties of interleukin-1. Eur Cytokine Netw 5: 517-531, 1994.

53. Sims J, Towne J and Blumberg H: IL-1 family members in inflammatory skin disease. Ernst Schering Res Found Workshop 56: $187-191,2006$

54. Paquet $\mathrm{P}$ and Pierard GE: Interleukin-6 and the skin. Int Arch Allergy Immunol 109: 308-317, 1996.

55. Hernandez-Quintero M, Kuri-Harcuch W, Gonzalez Robles A and Castro-Munozledo F: Interleukin-6 promotes human epidermal keratinocyte proliferation and keratin cytoskeleton reorganization in culture. Cell Tissue Res 325: 77-90, 2006. Epub ahead of print March 21, 2006.

56. Brauchle M, Angermeyer K, Hubner G and Werner S: Large induction of keratinocyte growth factor expression by serum growth factors and pro-inflammatory cytokines in cultured fibroblasts. Oncogene 9: 3199-3204, 1994.

57. Fehgali CA, Bost KL, Boulware DW and Levy LS: Human recombinant interleukin-4 induces proliferation and interleukin-6 production by cultured human skin fibroblasts. Clin Immunol Immunopathol 63: 182-187, 1992.

58. Mann A, Breuhahn K, Schirmacher P and Blessing M: Keratinocyte-derived granulocyte-macrophage colony stimulating factor accelerates wound healing: Stimulation of keratinocyte proliferation, granulation tissue formation, and vascularization. J Invest Dermatol 117: 1382-1390, 2001

59. Trent JT and Kerdel FA: Tumor necrosis factor alpha inhibitors for the treatment of dermatologic diseases. Dermatol Nurs 17 : 97-107, 2005

60. Faler BJ, Macsata RA, Plummer D, Mishra L and Sidawy AN: Transforming growth factor-beta and wound healing. Perspect Vasc Surg Endovasc Ther Mar 18: 55-61, 2006.

61. Leask A and Abraham DJ: TGF-beta signaling and the fibrotic response. FASEB J 18: 816-827, 2004.
62. Kirsner RS, Falanga V and Eaglstein WH: The biology of skin grafts. Skin grafts as pharmacologic agents. Arch Dermatol 129: 481-483, 1993.

63. Heneine W, Tibell A, Switzer W, Sandstrom P, Rosales GV, Mathews A, Korsgren O, Chapman LE, Folks TM and Groth CG: No evidence of infection with porcine endogenous retrovirus in recipients of porcine islet-cell xenografts. Lancet 352: 695-699, 1998.

64. Kagan RJ: FDA regulation of human tissue: implications for skin banks and burn centers. J Burn Care Rehabil 21: 353-357, 2000.

65. Parravicini C, Olsen SJ, Capra M, Poli F, Sirchia G, Gao SJ, Berti E, Nocera A, Rossi E, Bestetti G, Pizzuto M, Galli M, Moroni M, Moore PS and Corbellino M: Risk of Kaposi's sarcoma-associated herpes virus transmission from donor allografts among Italian posttransplant Kaposi's sarcoma patients. Blood 90: 2826-2829, 1997.

66. Stramer SL, Glynn SA, Kleinman SH, Strong DM, Caglioti S, Wright DJ, Dodd RYand Busch MP; National Heart, Lung, and Blood Institute Nucleic Acid Test Study Group: Detection of HIV-1 and HCV infections among antibody-negative blood donors by nucleic acid-amplification testing. N Engl J Med 351: 760-768, 2004.

67. Mohan J, Brown KL, Farquhar CF, Bruce ME and Mabbott NA Scrapie transmission following exposure through the skin is dependent on follicular dendritic cells in lymphoid tissues. J Dermatol Sci 35: 101-111, 2004.

68. Lomas RJ, Jennings LM, Fisher J and Kearney JN: Effects of a peracetic acid disinfection protocol on the biocompatibility and biomechanical properties of human patellar tendon allografts. Cell Tissue Bank 5: 149-160, 2004.

69. Eastlund T: Infectious disease transmission through cell, tissue, and organ transplantation: Reducing the risk through donor selection. Cell Transplant 4: 455-477, 1995

70. Goddard MJ, Foweraker JE and Wallwork J: Xenotransplantation-2000. J Clin Pathol 53: 44-48, 2000.

71. Weiss RA: Xenografts and retroviruses. Science 285: 1221-1222, 1999.

72. Patience C, Patton GS, Takeuchi Y, Weiss RA, McClure MO, Rydberg L and Breimer ME: No evidence of pig DNA or retroviral infection in patients with short-term extracorporeal connection to pig kidneys. Lancet 352: 699-701, 1998.

73. Pitkin Z and Mullon C: Evidence of absence of porcine endogenous retrovirus (PERV) infection in patients treated with a bioartificial liver support system. Artif Organs 23: 829-833, 1999.

74. Elliott RB, Escobar L, Garkavenko O, Croxson MC, Schroeder BA, McGregor M, Ferguson G, Beckman N and Ferguson S: No evidence of infection with porcine endogenous retrovirus in recipients of encapsulated porcine islet xenografts. Cell Transplant 9: 895-901, 2000.

75. Dinsmore JH, Manhart C, Raineri R, Jacoby DB and Moore A: No evidence for infection of human cells with porcine endogenous retrovirus (PERV) after exposure to porcine fetal neuronal cells. Transplantation 70: 1382-1389, 2000.

76. Levy MF, Crippin J, Sutton S, Netto G, McCormack J, Curiel T, Goldstein RM, Newman JT, Gonwa TA, Banchereau J, Diamond LE, Byrne G, Logan J and Klintmalm GB: Liver allotransplantation after extracorporeal hepatic support with transgenic (hCD55/hCD59) porcine livers: clinical results and lack of pig-to-human transmission of the porcine endogenous retrovirus. Transplantation 69: 272-280, 2000.

77. Xu H, Sharma A, Okabe J, Cui C, Huang L, Wei YY, Wan H, Lei Y, Logan JS, Levy MF and Byrne GW: Serologic analysis of anti-porcine endogenous retroviruses immune responses in humans after ex vivo transgenic pig liver perfusion. ASAIO J 49: 407-416, 2003.

78. Magre S, Takeuchi $Y$ and Bartosch B: Xenotransplantation and pig endogenous retroviruses. Rev Med Virol 13: 311-329, 2003.

79. Irgang M, Sauer IM, Karlas A, Zeilinger K, Gerlach JC, Kurth R, Neuhaus P and Denner J: Porcine endogenous retroviruses: no infection in patients treated with a bioreactor based on porcine liver cells. J Clin Virol 28: 141-154, 2003.

80. Denner J: Porcine endogenous retroviruses (PERVs) and xenotransplantation: screening for transmission in several clinical trials and in experimental models using non-human primates. Ann Transplant 8: 39-48, 2003

81. McKane BW, Ramachandran S, Xu XC, Olack BJ, Chapman WC and Mohanakumar T: Natural antibodies prevent in vivo transmission of porcine islet-derived endogenous retrovirus to human cells. Cell Transplant 13: 137-143, 2004.

82. Fishman JA and Patience C: Xenotransplantation: Infectious risk revisited. Am J Transplant 4: 1383-1390, 2004. 
83. Boneva RS and Folks TM: Xenotransplantation and risks of zoonotic infections. Ann Med 36: 504-517, 2004.

84. Di Nicuolo G, van de Kerkhove MP, Hoekstra R, Beld MG, Amoroso P, Battisti S, Starace M, di Florio E, Scuderi V, Scala S, Bracco A, Mancini A, Chamuleau RA and Calise F: No evidence of in vitro and in vivo porcine endogenous retrovirus infection after plasmapheresis through the AMC-bioartificial liver. Xenotransplantation 12: 286-292, 2005.

85. Winkler ME, Winkler M, Burian R, Hecker J, Loss M, Przemeck M, Lorenz R, Patience C, Karlas A, Sommer S, Denner J and Martin U: Analysis of pig-to-human porcine endogenous retrovirus transmission in a triple-species kidney xenotransplantation model. Transpl Int 17: 848-858, 2005. Epub April 29, 2005.
86. Karlas A, Kurth R and Denner J: Inhibition of porcine endogenous retroviruses by RNA interference: increasing the safety of xenotransplantation. Virology 325: 18-23, 2004.

87. Miyagawa S, Nakatsu S, Nakagawa T, Kondo A, Matsunami K, Hazama K, Yamada J, Tomonaga K, Miyazawa T and Shirakura R: Prevention of PERV infections in pig to human xenotransplantation by the RNA interference silences gene. J Biochem 137: 503-508, 2005.

88. Miyagawa S, Nakatsu S, Hazama K, Nakagawa T, Kondo A, Matsunami K, Yamamoto A, Yamada J, Miyazawa T and Shirakura R: A novel strategy for preventing PERV transmission to human cells by remodeling the viral envelope glycoprotein. Xenotransplantation 13: 258-263, 2006. 